\title{
Retraction: Effects of Large-Angle Coulomb Collisions on Inertial Confinement Fusion Plasmas [Phys. Rev. Lett. 112, 245002 (2014)]
}

\author{
A. E. Turrell, M. Sherlock, and S. J. Rose \\ (Received 2 September 2014; published 26 September 2014)
}

DOI: 10.1103/PhysRevLett.113.139904

PACS numbers: 52.25.Fi, 52.57.-z, 52.65.Pp, 99.10.Ln

Shortly after the publication of this Letter, it was brought to our attention that the results describing an increase in the rate of temperature equilibration of electrons and ions due to the inclusion of large-angle collisions in the $2 \lesssim \ln \Lambda \lesssim 5$ regime were in conflict with the results of molecular dynamics (MD) simulations of the rate of temperature equilibration between electrons and ions [1-3]. While trying to understand this difference, an error was discovered in the Monte Carlo code in which the small-angle Coulomb logarithm was incorrectly set when large-angle collisions were included in simulations.

This error causes the strength of small-angle Coulomb collisions to be overestimated when large-angle Coulomb collisions are included. The removal of this error significantly affects the results on electron-ion temperature equilibration; with the error corrected, the increase in the rate of equilibration is no longer present. As this error affects only the smallangle Coulomb collisions, we expect that our results on the generation of knock-on ions, and the distortion away from Maxwell-Boltzmann distribution functions that they cause, to be broadly correct. However, the main conclusion of the Letter concerned the increase in electron-ion temperature equilibration due to large-angle collisions. As this increase has been found to be erroneous, we have decided to retract the Letter.

We thank Andris Dimits, and others at Lawrence Livermore National Laboratory, for contacting us about the discrepancy between our results and those of MD simulations which led to our discovery of the coding error.

[1] G. Dimonte and J. Daligault, Phys. Rev. Lett. 101, 135001 (2008).

[2] J. N. Glosli, F. R. Graziani, R. M. More, M. S. Murillo, F. H. Streitz, M. P. Surh, L. X. Benedict, S. Hau-Riege, A. B. Langdon, and R. A. London, Phys. Rev. E 78, 025401 (2008).

[3] L. X. Benedict, J. N. Glosli, D. F. Richards, F. H. Streitz, S. P. Hau-Riege, R. A. London, F. R. Graziani, M. S. Murillo, and J. F. Benage, Phys. Rev. Lett. 102, 205004 (2009).

Published by the American Physical Society under the terms of the Creative Commons Attribution 3.0 License. Further distribution of this work must maintain attribution to the author(s) and the published articles title, journal citation, and DOI. 